\title{
Controlled functionalization of graphene oxide with sodium azide $\uparrow$
}

Cite this: Nanoscale, 2013, 5, 12136

\author{
Siegfried Eigler, ${ }^{\text {*a }}$ Yichen Hu, ${ }^{\text {b }}$ Yoshitaka Ishii ${ }^{\text {bc }}$ and Andreas Hirsch ${ }^{a}$
}

Received 15th August 2013

Accepted 10th October 2013

DOI: $10.1039 / c 3 n r 04332 k$

www.rsc.org/nanoscale

We present the first example of azide functionalization on the surface of graphene oxide (GO), which preserves thermally unstable groups in GO through the mild reaction with sodium azide in solids. Experimental evidence, by ${ }^{15} \mathrm{~N}$ solid-state NMR and other spectroscopic methods, indicates the substitution of organosulfate with azide anions as the reaction mechanism.

Graphene oxide (GO) is a nanomaterial that has attracted interdisciplinary attention for its broad applications across the fields of physics, chemistry and medicine $\cdot{ }^{1-4}$ It can be prepared from graphite in large amounts and processed from solution. ${ }^{5}$ The treatment of GO by thermal methods or by reducing agents yields an electrically conductive graphene-based material. ${ }^{6}$ Furthermore, single layers of graphene with tunable defect density and electronic properties can be obtained from $\mathrm{GO}^{7,8}$

Despite its great promise, chemical functionalization of GO is still in its early stage, and novel chemical tools for the modification of GO are demanded. ${ }^{9}$ For example, new molecular architectures of graphene- or GO-based materials are highly desired in the field of sensors or to alter the electronic properties of graphene. ${ }^{10,11}$ Among a variety of functionalization methods for graphene-based materials, the use of nitrogen containing reagents has attracted great attention. Hydrazine has been utilized to reduce $\mathrm{GO}$ to restore $\mathrm{sp}^{2}$ conjugation. ${ }^{12}$ Production of high quality N-doped graphene was achieved in

${ }^{a}$ Department of Chemistry and Pharmacy, Institute of Advanced Materials and Processes (ZMP), Friedrich-Alexander-Universität Erlangen-Nürnberg (FAU), Dr-Mack Str. 81, 90762 Fürth, Germany. E-mail: siegfried.eigler@fau.de; Fax: +49 (0)911 6507865015; Tel: +49 (0)911 6507865005

${ }^{b}$ Department of Chemistry, University of Illinois at Chicago, $845 \mathrm{~W}$ Taylor St, Chicago, IL 60607, USA

${ }^{c}$ Center for Structural Biology, University of Illinois at Chicago, 1100 South Ashland Street, Chicago, Illinois 60607, USA

$\dagger$ Electronic supplementary information (ESI) available: General methods, solid-state NMR analysis, ab initio calculations, preparation procedures, FTIR analysis of GO films on ZnSe, and detailed TG-MS analysis. See DOI: $10.1039 / \mathrm{c} 3 \mathrm{nr} 04332 \mathrm{k}$ recent years by growing graphene using chemical vapor deposition using ammonia as the doping source. ${ }^{11,13} \mathrm{~N}$-doping within the carbon framework of graphene highly improves probing of organic molecules by graphene-enhanced Raman spectroscopy. ${ }^{13}$ Approaches using GO as a precursor for $\mathrm{N}$-doping lead to graphene that is rather $\mathrm{N}$-doped due to functionalization at edges of defects than within the carbon framework. ${ }^{14-16}$ These materials perform well for rewritable nonvolatile memories or n-type field effect transistors through reactions with a dimethylformamide or amino-benzene moiety. ${ }^{15,16}$ Nevertheless, one general drawback for making functionalized GO with these relatively reactive nitrogen containing reagents is its limited thermal stability and the dynamic change of GO's chemical structure in aqueous media. ${ }^{17-19}$ Therefore, GO decomposes in water, and permanent defects are introduced in an uncontrolled manner by the cleavage of the carbon framework. ${ }^{19}$ Thus, chemical functionalization, while preserving the carbon backbone, is one of the major challenges for preparing new GO-based molecular architectures with a controlled structure. Hence, we recently altered the synthetic protocol for the preparation of GO yielding a type of GO that exhibits an almost preserved carbon skeleton as a consequence of preventing the evolution of $\mathrm{CO}_{2}$ during synthesis. ${ }^{7}$ This material is highly reducible and the highest charge carrier mobility values exceeding $1000 \mathrm{~cm}^{2} \mathrm{~V}^{-1} \mathrm{~s}^{-1}$ could be measured. Further on, we realized that purified GO bears organosulfate groups on the basal plane as part of its chemical structure, in addition to epoxy and hydroxyl groups as described earlier. ${ }^{20-23}$

With this new type of GO in hand, here we introduce a novel synthetic path to functionalize GO in a controlled manner through selectively functionalizing the basal plane of GO with azide. This product azide-functionalized $\mathrm{GO}\left(\mathrm{GO}-\mathrm{N}_{3}\right)$ provides a versatile reactive motif for further chemical reactions such as "click" reactions or heterocycle formation. ${ }^{24}$ We also give insight into the reaction mechanism that involves substitution of organosulfate and a small number of oxides by azide groups (Scheme 1). ${ }^{20}$ Our study is based on complementary analysis including thermogravimetry coupled with mass spectrometry 


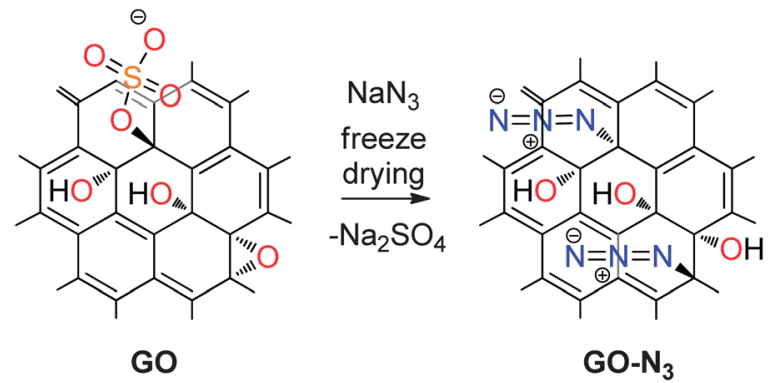

Scheme 1 Illustration of the reaction of sodium azide with GO; substitution of organosulfate and assumedly epoxy groups by azide.

(TG-MS), solid-state NMR (SSNMR) or Fourier transform infrared spectroscopy (FTIR). Importantly, we report that introducing azide at low temperature allows one to functionalize GO while largely preventing the decomposition of the basic structural frame of GO unlike previously proposed chemical or thermochemical reactions to modify GO. Furthermore, cleavage of $\mathrm{N}_{2}$ from azide followed by $\mathrm{N}$-insertion within the carbon $\sigma$-bonds is expected to be an entry to $\mathrm{N}$-doped graphene. Opening this field may result in new materials for charge storage devices, selective sensors or n-type transistors of high performance.

GO was prepared by a modified Hummers' method that prevents the $\sigma$-framework of carbon atoms from rupture on the few $\mathrm{nm}$ scale. ${ }^{7}$ Moreover, organosulfate groups in addition to epoxy and hydroxyl groups remain stable in aqueous solution at least for months if stored at $<10{ }^{\circ} \mathrm{C}^{20}$ Thus, we find a sulfur content of about $5 \%$ by elemental analysis as part of the structure of GO. At first, we investigated the reaction of GO by FTIR spectroscopy on ZnSe windows. The amount of sodium azide added to a dispersion of GO was altered between $8 \%$ and $42 \%$. As shown in Fig. $\mathrm{S} 1 \mathrm{~A} \dagger$ we find two absorptions at $2123 \mathrm{~cm}^{-1}$ and $2065 \mathrm{~cm}^{-1}$ that originate from the stretching mode of chemically bound azide to GO and excess sodium azide, respectively. With increasing excess of sodium azide the absorption at $2065 \mathrm{~cm}^{-1}$ develops. With respect to these results we tried to isolate $\mathrm{GO}-\mathrm{N}_{3}$ from solution by centrifugation. We purified the centrifuged material several times with water. Surprisingly, the final product did not show signals between 2000 and $2200 \mathrm{~cm}^{-1}$. From this experiment we concluded that the reaction does not proceed in solution but only in the solid state during drying of the dispersion on ZnSe used for FTIR spectroscopy. Because of that, a dispersion of GO and sodium azide was freeze-dried after mixing both using $0.8 \mathrm{mg}$ sodium azide and $1 \mathrm{mg}$ GO. After freeze-drying the material was purified by repeated centrifugation and redispersion in water and this time the reaction was successful. The final GO- $\mathrm{N}_{3}$ was freezedried again and the FTIR spectrum proves the azide functionalization as shown in Fig. 1A (black). Thus, we conclude that GO-N $\mathrm{N}_{3}$ is hydrolytically stable at room-temperature. To further confirm the origin of the FTIR absorption at $2123 \mathrm{~cm}^{-1}$ we repeated the functionalization experiment using $\mathrm{Na}^{15} \mathrm{~N}^{14} \mathrm{~N}_{2}$ and found a $11 \mathrm{~cm}^{-1}$ shifted stretching vibration of azide at $2112 \mathrm{~cm}^{-1}$ Fig. 1A (red).

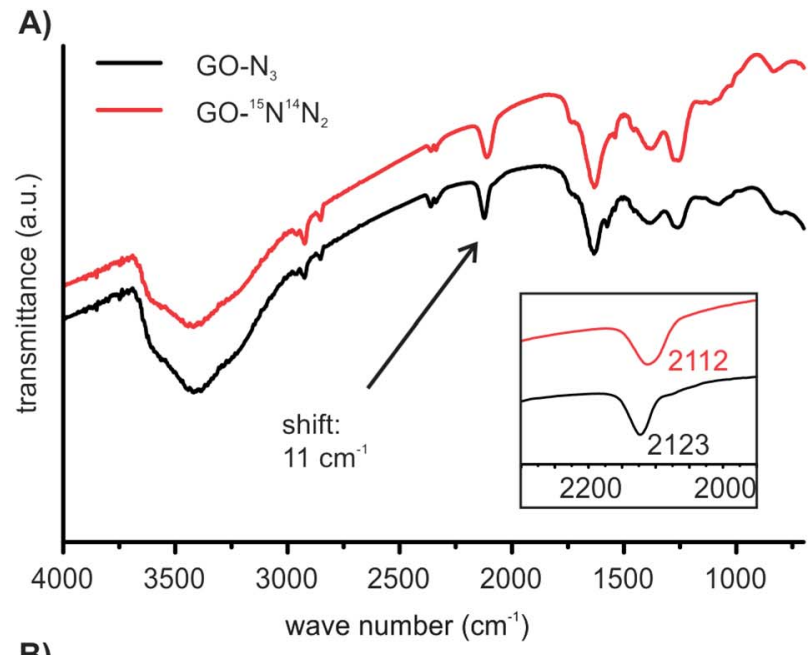

B)

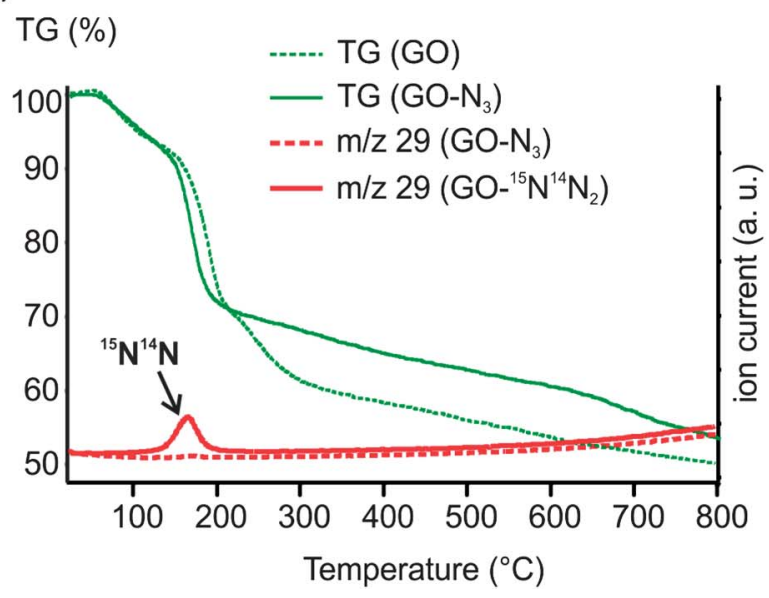

Fig. 1 (A) FTIR spectra of GO- $\mathrm{N}_{3}$ and $G O-{ }^{15} \mathrm{~N}^{14} \mathrm{~N}_{2}$; (B) TGA curve of $\mathrm{GO}$ and GO- ${ }^{15} \mathrm{~N}^{14} \mathrm{~N}_{2}, \mathrm{~m} / \mathrm{z} 29$ of GO-N $\mathrm{N}_{3}$ and GO- ${ }^{15} \mathrm{~N}^{14} \mathrm{~N}_{2}$

To give further evidence of the controlled mild functionalization of GO with azide we used TG-MS analysis. As shown in Fig. $1 \mathrm{~B}$ and $\mathrm{S} 2 \uparrow$ the main weight-loss step occurs between $150^{\circ} \mathrm{C}$ and $200{ }^{\circ} \mathrm{C}$. Water, $\mathrm{CO}, \mathrm{N}_{2}$, and $\mathrm{CO}_{2}$ are the main decomposition products that originate from hydroxyl and epoxy groups for both materials. To differentiate between $\mathrm{CO}$ and $\mathrm{N}_{2}$ in GO-N $\mathrm{N}_{3}$ we analyzed $\mathrm{GO}^{-15} \mathrm{~N}^{14} \mathrm{~N}_{2}$ as well and find a clear signal with $\mathrm{m} / \mathrm{z} 29$ for ${ }^{15} \mathrm{~N}^{14} \mathrm{~N}$ formed between 150 and $200{ }^{\circ} \mathrm{C}$. The total weigh-loss of GO- $\mathrm{N}_{3}$ and GO is about $30 \%$ up to $200{ }^{\circ} \mathrm{C}$, for both materials. This is an indication for GO- $\mathrm{N}_{3}$ being highly functionalized with oxygen carrying groups in contrast to earlier reports. ${ }^{25}$ However, in contrast to $\mathrm{GO}-\mathrm{N}_{3}$, the educt GO exhibits an additional weight-loss step above $200{ }^{\circ} \mathrm{C}$ that we recently assigned to the decomposition of organosulfate. ${ }^{20}$ This organosulfate can be identified in $\mathrm{GO}$ by the evolution of $\mathrm{SO}_{2}$ up to $300{ }^{\circ} \mathrm{C}$ during TGMS analysis $\left(\mathrm{m} / \mathrm{z}\right.$ 64, Fig. S2 $\dagger$ ). TG-MS analysis for GO-N $\mathrm{N}_{3}$ reveals no $\mathrm{SO}_{2}$ evolution.

These observations can be explained by the exchange of organosulfate with azide. To further prove this exchange we analyzed the supernatant after centrifugation of GO- $\mathrm{N}_{3}$ from the dispersed freeze dried $\mathrm{GO}-\mathrm{NaN}_{3}$ reaction mixture. We allowed the supernatant to dry in air and dissolved the residue in $1 \mathrm{M}$ 
$\mathrm{HCl}$. To verify the existence of sulfate we added $0.1 \mathrm{M} \mathrm{BaCl}_{2}$, filtered the precipitate, washed with water and dried it at $160{ }^{\circ} \mathrm{C}$. After that $\mathrm{BaSO}_{4}$ was identified by ATR-FTIR and the signature was compared to freshly precipitated $\mathrm{BaSO}_{4}$ prepared from $\mathrm{Na}_{2} \mathrm{SO}_{4}$ and $\mathrm{BaCl}_{2}$ (Fig. S3 $\dagger$ ). Elemental analyses of GO and GO$\mathrm{N}_{3}$ give insight into the proceeded reaction. On the one hand, the nitrogen content increases from $0.0 \%$ to $4.1 \%$ and on the other hand the sulfur content is reduced from $3.6 \%$ for GO to $1.7 \%$ for $\mathrm{GO}-\mathrm{N}_{3}$. The residual sulfur may originate from inorganic sulphate to some extent and is indicated by the weight-loss detected at about $700{ }^{\circ} \mathrm{C}$ (Fig. 2B). Using these data we estimate that there is about one azide group on 30 C-Atoms in GO- $\mathrm{N}_{3}$.

To find further evidence for the azide functionalization of GO we conducted ${ }^{15} \mathrm{~N}$ SSNMR analysis. This technique was effectively used to evaluate the structural properties of reduced GO that was obtained by the reaction of ${ }^{15} \mathrm{~N}$-labelled hydrazine and ${ }^{13} \mathrm{C}$-labeled GO. ${ }^{26}$ Fig. 2 illustrates (B) a ${ }^{15} \mathrm{~N}$ MAS SSNMR spectrum for GO- $\mathrm{N}_{3}$ prepared from $\mathrm{Na}^{15} \mathrm{~N}^{14} \mathrm{~N}_{2}$ and GO with (A) a simplified structural model of GO-N $\mathrm{N}_{3}$. The model shows, besides aromatic regions, a hydroxyl group adjacent to an azide group (trans-configuration). This model was obtained by geometry optimization using $a b$ initio calculations and used to simulate the resonance frequency of ${ }^{15} \mathrm{~N}$ SSNMR signals obtained for

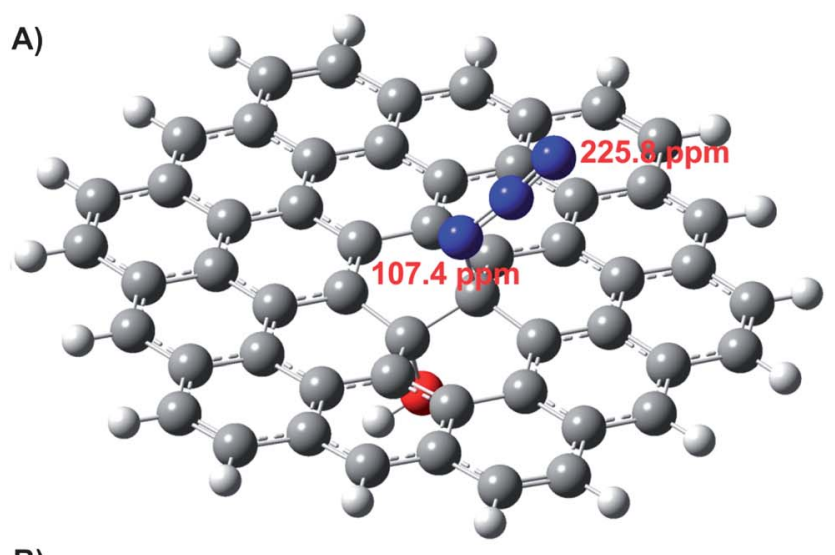

B)

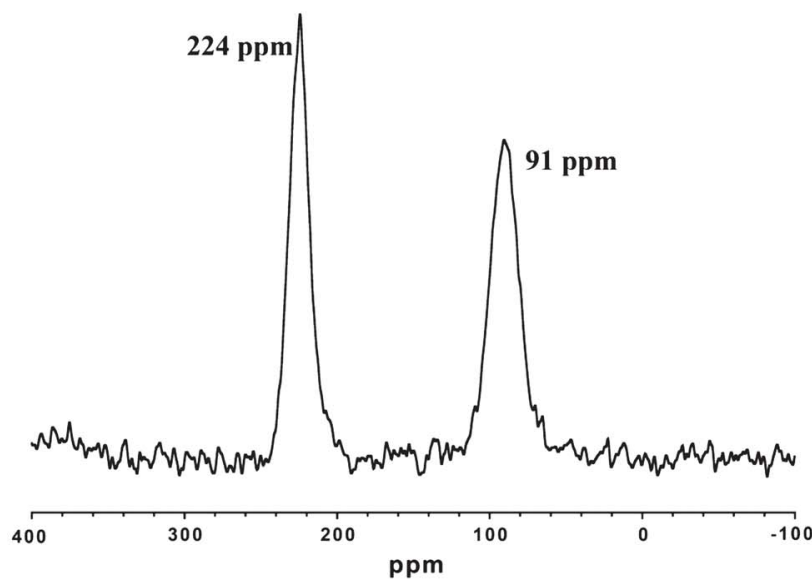

Fig. 2 (A) Simplified model of $\mathrm{GO}-\mathrm{N}_{3}$ with an azide and a hydroxyl group connected to the carbon lattice in trans-configuration, calculated by ab initio methods to predict ${ }^{15} \mathrm{~N}$ NMR shifts (107.4 ppm and $225.8 \mathrm{ppm}$ ); (B) ${ }^{15} \mathrm{~N}$ SSNMR MAS spectrum of GO- ${ }^{15} \mathrm{~N}^{14} \mathrm{~N}_{2}$ with two peaks (1: 1 ratio).
GO- ${ }^{15} \mathrm{~N}^{14} \mathrm{~N}_{2}$. The $a b$ initio geometry optimized structure showed no signs of instability. Since ${ }^{15} \mathrm{~N}$ in ${ }^{15} \mathrm{~N}^{14} \mathrm{~N}_{2}{ }^{-}$can be bound to GO in two configurations $\left(\mathrm{C}-{ }^{15} \mathrm{~N}-{ }^{14} \mathrm{~N}^{14} \mathrm{~N}\right.$ or $\left.\mathrm{C}-{ }^{14} \mathrm{~N}-{ }^{14} \mathrm{~N}-{ }^{15} \mathrm{~N}\right)$, two signals are expected if ${ }^{15} \mathrm{~N}^{14} \mathrm{~N}_{2}$ is chemically bound to GO. Consequently, two signals with an integral intensity ratio of $1: 1.05$ are obtained in the ${ }^{15} \mathrm{~N}$ SSNMR spectrum (Fig. $2 \mathrm{~B}$ ) for the two possible bounding configurations. No other peaks were experimentally confirmed. Furthermore, the ${ }^{15} \mathrm{~N}$ chemical shifts at $224 \mathrm{ppm}$ and $91 \mathrm{ppm}$ are consistent with ${ }^{15} \mathrm{~N}$ shifts predicted by the $a b$ initio calculations for the azide that is bound in end-on configuration (226 ppm) or with ${ }^{15} \mathrm{~N}$ being connected to the $\mathrm{sp}^{3}$ carbon atom (107 ppm). These results are an excellent indication for the controlled functionalization of GO with azide without formation of any other nitrogen containing species as a major impurity incorporated into $\mathrm{N}_{3}$-GO.

All the analytical results indicate that a large amount of organosulfate becomes accessible for azide to be substituted (Scheme 1). Since azide is a good anionic nucleophile and anionic organosulfate is present on both sides of GO's carbon backbone, a reaction in solution is hindered by steric effects and especially due to electrostatic repulsion. Thus, this is an explanation why the reaction of sodium azide and GO was conducted at high temperature and why long reaction times had been necessary causing significant decomposition as reported by Salvio et $a ._{.}{ }^{25}$ Furthermore, Salvio et al. proposed "click" reactions, which however proceed rather at edges than on the surface of GO. Such chemistry is not effective to develop GO based materials with minimal defects. Whereas, in the solid state the situation is different and since azide is adsorbed on the surface of GO the local azide concentration is very high and therefore, organosulfate can now react as a leaving group assuming a nucleophilic reaction. Besides, we assume that few epoxy groups can act as reaction partners as well, as indicated in Scheme 1. Finally, we tested the thermal stability of GO- $\mathrm{N}_{3}$ both in solids and in aqueous dispersion (Fig. S4 $\dagger$ ). In solids we find that degradation of GO- $\mathrm{N}_{3}$ starts at about $80{ }^{\circ} \mathrm{C}$. The degradation is indicated by $\mathrm{CO}_{2}$ formation and the $\mathrm{CO}_{2}$ is trapped between layers of the GO-N $\mathrm{N}_{3}$ film. This trapped $\mathrm{CO}_{2}$ is analytically accessible for FTIR analysis (Fig. S4A $\dagger$ ). ${ }^{18}$ At higher temperature azide bound to GO degrades in agreement with TGMS analysis (Fig. 1B). Next, GO-N $\mathrm{N}_{3}$ was treated for $30 \mathrm{~min}$ at a certain temperature with water. We find that azide in GO-N $\mathrm{N}_{3}$ is cleaved in aqueous dispersion starting at $80{ }^{\circ} \mathrm{C}$ and especially at $100{ }^{\circ} \mathrm{C}$, as indicated by the FTIR vibration at $2058 \mathrm{~cm}^{-1}$ (Fig. S4B $\dagger$ ). After $16 \mathrm{~h}$ of treatment little degradation is indicated for the $60{ }^{\circ} \mathrm{C}$ sample, and for the $80{ }^{\circ} \mathrm{C}$ sample decomposition is detected (Fig. S5 $\dagger$ ). These results reveal that GO-N $\mathrm{N}_{3}$ is hydrolytically stable in a range between room temperature and $60{ }^{\circ} \mathrm{C}$, which has been utilized in azide-based chemistry such as "click" reactions. Thus, thermal activation can be used when adopting GO-N $\mathrm{N}_{3}$ as a precursor for other reactions as long as attention is paid to the compound stability.

\section{Conclusions}

We successfully established the direct functionalization of GO with $\mathrm{N}_{3}$ preserving functional groups in $\mathrm{GO}$ for the first time. 
Our analysis is based on FTIR, TG-MS, EA and ${ }^{15} \mathrm{~N}$ SSNMR to prove the successful functionalization of GO using reactions at low temperature in solids. This new method preserves thermally unstable groups present in GO as indicated by TG-MS analysis. Moreover, azide is predominately located on the surface of GO and not at edges. Although edge structures of GO involving chemical species such as carbonyl and carboxyl groups have been reported, ${ }^{27}$ the possibility of the reactions of such edge species with azide will be examined in our future studies. Based on our findings we estimate approximately one azide group on 30 carbon atoms. Further on, the azide group in GO-N $\mathrm{N}_{3}$ is hydrolytically stable up to $60{ }^{\circ} \mathrm{C}$ and therefore we believe that GO- $\mathrm{N}_{3}$ is a suitable precursor for other reactions, opening an avenue for the field of azide chemistry for graphene-based materials. Further development will lead to $n$-doped graphene, amine functionalized graphene after reduction or positively charged graphene after quarterisation. Besides, "click" reactions and Staudinger reactions become feasible to generate molecular architectures while the basic structure of GO is preserved. Further development of mild reactions with GO is highly demanded to find an access to highly conductive and functionalized graphene. Such new structures may e.g. make new sensor materials possible based on GO- $\mathrm{N}_{3}$ that selectively bind substrates and thus potentially enhance the field of medical applications. Another possible research field may be the development of $n$-type transistors with enhanced charge carrier mobility values.

\section{Acknowledgements}

The authors thank the Deutsche Forschungsgemeinschaft (DFG-SFB 953, Project A1 "Synthetic Carbon Allotropes"), the European Research Council (ERC; grant 246622 - GRAPHENOCHEM), and the Cluster of Excellence 'Engineering of Advanced Materials (EAM)' for financial support. The solid-state NMR analysis was supported by funding from the U.S. National Science Foundation (NSF; CHE 957793, CHE 1310363) for YI. The purchase of the NMR instrument used in this study was, in part, supported by the NSF (CHE 957793), the U.S. Department of Energy Basic Sciences (DE-SC001951), and the U.S. National Institutes of Health (GM098033).

\section{Notes and references}

1 V. Georgakilas, M. Otyepka, A. B. Bourlinos, V. Chandra, N. Kim, K. C. Kemp, P. Hobza, R. Zboril and K. S. Kim, Chem. Rev., 2012, 112, 6156-6214.

2 K. S. Novoselov, V. I. Fal'ko, L. Colombo, P. R. Gellert, M. G. Schwab and K. Kim, Nature, 2012, 490, 192200.

3 C. Chung, Y. K. Kim, D. Shin, S. R. Ryoo, B. H. Hong and D. H. Min, Acc. Chem. Res., 2013, 46, 2211-2224.

4 H. Y. Mao, S. Laurent, W. Chen, O. Akhavan, M. Imani, A. A. Ashkarran and M. Mahmoudi, Chem. Rev., 2013, 113, 3407-3424.
5 D. A. Dikin, S. Stankovich, E. J. Zimney, R. D. Piner, G. H. B. Dommett, G. Evmenenko, S. T. Nguyen and R. S. Ruoff, Nature, 2007, 448, 457-460.

6 S. Pei and H.-M. Cheng, Carbon, 2012, 50, 3210-3228.

7 S. Eigler, M. Enzelberger-Heim, S. Grimm, P. Hofmann, W. Kroener, A. Geworski, C. Dotzer, M. Rockert, J. Xiao, C. Papp, O. Lytken, H. P. Steinruck, P. Muller and A. Hirsch, Adv. Mater., 2013, 25, 3583-3587.

8 S. Eigler, S. Grimm, M. Enzelberger-Heim, P. Muller and A. Hirsch, Chem. Commun., 2013, 49, 7391-7393.

9 D. Chen, H. Feng and J. Li, Chem. Rev., 2012, 112, 6027-6053. 10 T. Kuila, S. Bose, P. Khanra, A. K. Mishra, N. H. Kim and J. H. Lee, Biosens. Bioelectron., 2011, 26, 4637-4648.

11 L. Zhao, R. He, K. T. Rim, T. Schiros, K. S. Kim, H. Zhou, C. Gutierrez, S. P. Chockalingam, C. J. Arguello, L. Palova, D. Nordlund, M. S. Hybertsen, D. R. Reichman, T. F. Heinz, P. Kim, A. Pinczuk, G. W. Flynn and A. N. Pasupathy, Science, 2011, 333, 999-1003.

12 S. Park and R. S. Ruoff, Nat. Nanotechnol., 2009, 4, 217-224. 13 R. Lv, Q. Li, A. R. Botello-Mendez, T. Hayashi, B. Wang, A. Berkdemir, Q. Hao, A. L. Elias, R. Cruz-Silva, H. R. Gutierrez, Y. A. Kim, H. Muramatsu, J. Zhu, M. Endo, H. Terrones, J. C. Charlier, M. Pan and M. Terrones, Sci. Rep., 2012, 2, 586.

14 T. Palaniselvam, H. B. Aiyappa and S. Kurungot, J. Mater. Chem., 2012, 22, 23799-23805.

15 S. Seo, Y. Yoon, J. Lee, Y. Park and H. Lee, ACS Nano, 2013, 7, 3607-3615.

16 D. W. Chang, E. K. Lee, E. Y. Park, H. Yu, H. J. Choi, I. Y. Jeon, G. J. Sohn, D. Shin, N. Park, J. H. Oh, L. Dai and J. B. Baek, J. Am. Chem. Soc., 2013, 135, 8981-8988.

17 S. Kim, S. Zhou, Y. Hu, M. Acik, Y. J. Chabal, C. Berger, W. de Heer, A. Bongiorno and E. Riedo, Nat. Mater., 2012, 11, 544549.

18 S. Eigler, C. Dotzer, A. Hirsch, M. Enzelberger and P. Müller, Chem. Mater., 2012, 24, 1276-1282.

19 A. M. Dimiev, L. B. Alemany and J. M. Tour, ACS Nano, 2013, 7, 576-588.

20 S. Eigler, C. Dotzer, F. Hof, W. Bauer and A. Hirsch, Chem.Eur. J., 2013, 19, 9490-9496.

21 A. Lerf, H. Heb, T. Riedl, M. Forster and J. Klinowskib, Solid State Ionics, 1997, 101-103, 857-862.

22 W. Cai, R. D. Pine, F. Stadermann, S. Park, M. Shaibat, Y. Ishii, D. Yang, A. Velamakanni, S. J. An, M. Stoller, J. An, D. M. Chen and R. S. Ruoff, Science, 2008, 321, 1815-1818.

23 L. B. Casabianca, M. Shaibat, W. Cai, S. Park, R. Piner, R. S. Ruoff and Y. Ishii, J. Am. Chem. Soc., 2010, 132, 5672-5676.

24 S. Bräse, C. Gil, K. Knepper and V. Zimmermann, Angew. Chem., Int. Ed., 2005, 44, 5188-5240.

25 R. Salvio, S. Krabbenborg, W. J. Naber, A. H. Velders, D. N. Reinhoudt and W. G. van der Wiel, Chem.-Eur. J., 2009, 15, 8235-8240.

26 S. Park, Y. Hu, J. O. Hwang, E.-S. Lee, L. B. Casabianca, W. Cai, J. R. Potts, H.-W. Ha, S. Chen, J. Oh, S. O. Kim, Y.-H. Kim, Y. Ishii and R. S. Ruoff, Nat. Commun., 2012, 3, 638.

27 D. R. Dreyer, S. Park, C. W. Bielawski and R. S. Ruoff, Chem. Soc. Rev., 2010, 39, 228-240. 